\title{
Identifying optimal candidates for liver resection or transarterial chemoembolisation in patients with unresectable hepatocellular carcinoma
}

\author{
Shoujie Zhao ${ }^{1 \#}$, Xiangnan Zhang ${ }^{2 \#}$, Mengmeng Wang ${ }^{3 \#}$, Kai Tan ${ }^{1 \#}$, Weijia Dou ${ }^{4}$, Qingling Fan ${ }^{4}$, \\ Huichen $\mathrm{Li}^{5}$, Xilin $\mathrm{Du}^{1}$, Lei Liu ${ }^{4}$ \\ ${ }^{1}$ Department of General Surgery, ${ }^{2}$ Division of Scientific Research, Tangdu Hospital, Fourth Military Medical University, Xi'an 710038, China; \\ ${ }^{3}$ Department of Drug and Equipment, Aeromedicine Identification and Training Centre of Air Force, Xi'an 710069, China; ${ }^{4}$ Department of \\ Gastroenterology, Tangdu Hospital, Fourth Military Medical University, Xi'an 710038, China; ${ }^{5}$ The State Key Laboratory of Cancer Biology, \\ Department of Biochemistry and Molecular Biology, School of Basic Medicine, Fourth Military Medical University, Xi'an 710032, China \\ Contributions: (I) Conception and design: L Liu; (II) Administrative support: H Li; (III) Provision of study materials or patients: Q Fan; (IV) \\ Collection and assembly of data: W Dou; (V) Data analysis and interpretation: M Wang, X Zhang, S Zhao; (VI) Manuscript writing: All authors; (VII) \\ Final approval of manuscript: All authors. \\ \#These authors contributed equally to this work. \\ Correspondence to: Lei Liu, MD, PhD. Department of Gastroenterology, Tangdu Hospital of the Fourth Military Medical University 569 Xinsi Road, \\ Xi'an 710038, China. Email: tdliulei@fmmu.edu.cn; Xilin Du, MD, PhD. Department of General Surgery,Tangdu Hospital of Fourth Military \\ Medical University 569 Xinsi Road, Xi’an 710038, China. Email: dxlin0705@163.com.
}

Background: Recommended as the first-line treatment for advanced unresectable hepatocellular carcinoma (HCC), sorafenib has been shown to prolong median overall survival (OS) for patients. However, advanced HCC sees high heterogeneity across patient groups. Recently, a growing number of studies have indicated surgical resection and transarterial chemoembolisation (TACE) to perform well in patients with portal vein tumor thrombosis (PVTT). The aim of this study was to compare the outcomes of liver resection and TACE and to identify prognostic factors related to OS for BCLC stage C patients with performance status (PS) 1 who have a single tumor but no vascular invasion or extrahepatic spread.

Methods: A total of 323 consecutive patients in BCLC stage C with PS 1 who had only one tumor and no vascular invasion or extrahepatic spread were enrolled in this retrospective study, regardless of tumor size. Survival analyses were performed using the Kaplan-Meier analysis, and statistical differences between the TACE and sorafenib groups were examined by the log-rank test. Univariate and multivariate Cox regression analyses were performed to investigate the prognostic factors for OS.

Results: Based on the Kaplan-Meier curves, patients treated with surgical resection showed a better OS than those who underwent TACE, with OS at 1, 3, and 5 years $(85.7 \%, 48.8 \%$, and $33.3 \%$ vs. $66.6 \%, 21.8 \%$, and $13.4 \%$, respectively; log-rank $\mathrm{P}<0.001$ ). Univariate and multivariate analyses demonstrated that tumor size, albumin, bilirubin, Child-Pugh score, and treatment method were significant prognostic factors for OS. According to the subgroup analyses based on tumor size, there were significant differences in OS among overall subsets between patients who underwent hepatectomy and those who underwent TACE therapy.

Conclusions: Liver resection had a better prognostic performance than TACE and should be put forward as an alternative treatment modality to TACE for BCLC stage C patients with PS 1 who have a single tumor and no vascular invasion or extrahepatic spread.

Keywords: Liver resection; transarterial chemoembolisation; hepatocellular carcinoma (HCC); eastern cooperative oncology group (ECOG)

Submitted Jan 08, 2020. Accepted for publication Feb 10, 2020.

doi: $10.21037 /$ atm.2020.02.83

View this article at: http://dx.doi.org/10.21037/atm.2020.02.83

(c) Annals of Translational Medicine. All rights reserved. 


\section{Introduction}

Liver cancer ranks as the seventh most common primary malignant tumor and is the third leading cause of cancerrelated mortality around the world (1). Hepatocellular carcinoma (HCC) accounts for more than $90 \%$ of liver cancer cases (2). The Barcelona Clinic Liver Cancer (BCLC) staging system holds a prominent advantage for predicting prognosis and has been widely adopted in clinical practice for allocating treatment $(3,4)$. On account of HCC's dormant and asymptomatic characteristics, a significant proportion of patients are already at the advanced stage of the disease by the time they receive their diagnosis and are beyond the optimal indication for curative treatments such as hepatectomy, liver transplantation, and radiofrequency ablation (5).

Patients with symptomatic tumors [performance status (PS), 1-2], vascular invasion, or extrahepatic spread who are classified as BCLC stage $\mathrm{C}$ have a dismal prognosis, with an expected median overall survival (OS) of 6-8 months (2). Sorafenib, an orally administered multikinase inhibitor, has been put forward as the first-line treatment for patients with advanced unresectable HCC and has been shown to prolong their median OS $(6,7)$.

The BCLC staging classification recommends liver resection and transarterial chemoembolisation (TACE) for treating early- to intermediate-stage HCC patients (2). Both treatments have been verified to improve survival outcomes, particularly when they were received in combination with other therapies including radiofrequency ablation, sorafenib and stereotactic body radiation therapy in several studies (8-10). With the development of treatment modalities and perioperative management, aggressive therapies (including but not limited to liver resection, TACE, and radiofrequency ablation) are no longer contraindications, instead offering alternative treatment options for patients with advanced HCC $(11,12)$. Notably, a growing number of studies have advocated the application of liver resection and TACE in patients with portal vein invasion $(13,14)$.

Eastern Cooperative Oncology Group performance status (ECOG-PS) is commonly used to stratify HCC stage and to select appropriate treatment decisions for patients in the BCLC system (15). Additionally, PS has been demonstrated to be an independent prognostic indicator of HCC patients at each stage who are undergoing different treatment modalities (16-18).

Despite published studies having explored liver resection and TACE in treating advanced HCC, whether liver resection is more beneficial than TACE for BCLC stage C patients with PS 1 who are without vascular invasion or extrahepatic spread has not yet been fully elucidated.

The present study aimed to compare the outcomes of liver resection and TACE. In addition, we identified prognostic factors related to OS for BCLC stage C patients with PS 1 who had no vascular invasion or extrahepatic spread.

\section{Methods}

\section{Study design and participants}

This study was conducted retrospectively and included consecutive HCC patients who underwent liver resection or TACE therapy at our department in the period from January 2010 to December 2017. The inclusion criteria for this study were as follows: (I) patients classified as BCLC stage $\mathrm{C}$ with a single tumor; (II) no vascular invasion or extrahepatic spread; (III) ECOG PS score 1; (IV) no previous therapy for HCC. Patients who met any of the following criteria were excluded from the study: (I) patients with other uncontrolled ascites, hepatic encephalopathy, or simultaneous malignancies of other system; (II) patients with cardiopulmonary, renal, or cerebral dysfunction.

HCC was diagnosed by contrast-enhanced magnetic resonance imaging (MRI) and computed tomography (CT) according to the guidelines of the American Association for the Study of the Liver Disease or the European Association for the Study of Liver disease (AASLD/EASL) $(19,20)$. Clinical, laboratory, and imaging data of enrolled patients were collected from hospital database. Because of the study's retrospective nature, the requirement to obtain informed consent was waived. This investigation was approved by the Clinical Research Ethics Committee of the Tangdu Hospital and performed in adherence with the Declaration of Helsinki.

\section{Treatment and follow-up}

Hepatectomy was conducted via a subcostal incision on the right side following general anesthesia being administered. The perihepatic ligaments and adhesion tissue were separated, before abdominal exploration was carried out. The number, size, distribution, and invasion of adjacent structures by liver tumors, as well as the relationship between tumors and vessels, bile ducts, and other structures were assessed using intraoperative ultrasound. Hepatic 
blood flow was blocked by applying the Pringle technique. The hepatoduodenal ligament was then clamped using a rubber tourniquet. According to the location, size, and number of the tumors, the patients underwent either hepatic lobectomy, hepatic segmentectomy, semi-hepatic resection, or partial resection. Each of the procedures were conducted in line with the standard methods recommended by the guidelines. All nodules were removed before being sent to the pathology department. All patients were followed up at one month after hepatectomy, and then every three months during the first year. Subsequently, patients were followed up as appropriate every three to six months.

For the TACE procedure, the Seldinger technique was used and a 4.1-French RC1 catheter was inserted into the tumor feeding artery. Following this, the number, location, size, and branches of the tumor feeding vessels were carefully identified. Then, an injection comprising $10-20 \mathrm{~mL}$ iodized oil, gelfoam particles with $30-50 \mathrm{mg}$ doxorubicin, and $50-100 \mathrm{mg}$ cisplatinum was administered via the arterial branches. Patients underwent TACE therapy every six weeks during the first year and every six to eight weeks thereafter, depending on their liver function and tumor necrosis.

Patients received routine examination at each followup, which included physical examinations, blood tests (serum $\alpha$-fetoprotein level, serum biochemistry, and liver biochemistry), and imaging examinations (chest X-ray, abdominal ultrasonography, and abdominal CT or MRI).

\section{Statistical analysis}

OS was defined as the time from the date of liver resection or TACE until death or the date of the last follow-up. The last visit took place on January, 2019. Categorical variables are expressed as frequencies, whereas continuous variables are expressed as medians and interquartile range. The baseline characteristics of enrolled patients in the two groups were compared using the chi-square test, or for categorical or continuous variables Fisher's exact test and Mann-Whitney U test were used, respectively. The KaplanMeier method was used to performed survival analyses, and the log-rank test was used to examine statistical differences between the liver resection and TACE groups. The Cox proportional hazards regression model was used to calculate the hazard ratio (HR) for survival and the $95 \%$ confidence intervals (CI) of prognostic factors for OS based on the univariate and multivariate analyses. To avoid collinearity, two multivariate models with stepwise methods were performed separately to select the independent prognostic factors: model 1, including the baseline characteristics but excluding the Child-Pugh score; and model 2, including the Child-Pugh score and baseline characteristics without albumin and bilirubin. Statistical significance was taken as a two-sided $\mathrm{P}$ value $\leq 0.05$ for all analyses. IBM SPSS software version 23.0 (SPSS Inc., Chicago, IL, USA) was used for all statistical analyses.

\section{Results}

\section{Baseline characteristics}

A total of 390 consecutive patients with BCLC stage C HCC who met the inclusion criteria were enrolled in this study. Of these, 204 and 186 patients comprised the liver resection group and TACE group, respectively. Based on the statistical analysis, no significant differences were observed in the characteristics between the two groups. The baseline demographic and clinical characteristics are summarized in Table 1.

\section{Survival analyses of patients in the liver resection and TACE groups}

The last follow-up for all included patients took place in October 2019. For those who underwent liver resection therapy, 119 patients died during a median follow-up period of 30.6 months. In TACE group, 71 patients died, while the median follow-up reached 32.8 months. Based on the Kaplan-Meier curves, the patients who underwent liver resection showed a better OS than those who underwent TACE, with OS of $83.2 \%, 60.8 \%, 33.3 \%$ vs. $66.6 \%, 25.2 \%$, $13.4 \%$ at 1,3 and 5 years, respectively (log-rank $\mathrm{P}<0.001$; Figure 1).

\section{Univariate and multivariate analyses of $O S$}

Based on the univariate analysis, tumor size, tumor number, treatment method, albumin, bilirubin, and Child-Pugh score were all factors associated with OS $(\mathrm{P}<0.05$; Table 2$)$. These factors were included in the multivariate analysis (Table 3). In multivariate model 1, tumor size (HR, 1.137; 95\% CI, 1.099-1.177; $\mathrm{P}<0.001$ ), bilirubin ( HR, 1.044; 95\% CI, 1.028-1.061; $\mathrm{P}<0.001)$, albumin (HR, 0.950; 95\% CI, 0.925-0.975; $\mathrm{P}<0.001$ ), and treatment method (HR, $2.610 ; 95 \% \mathrm{CI}, 1.935-3.521 ; \mathrm{P}<0.001)$ were identified as independent predictors of OS. In multivariate model 2, 
Table 1 The baseline characteristics of study patients

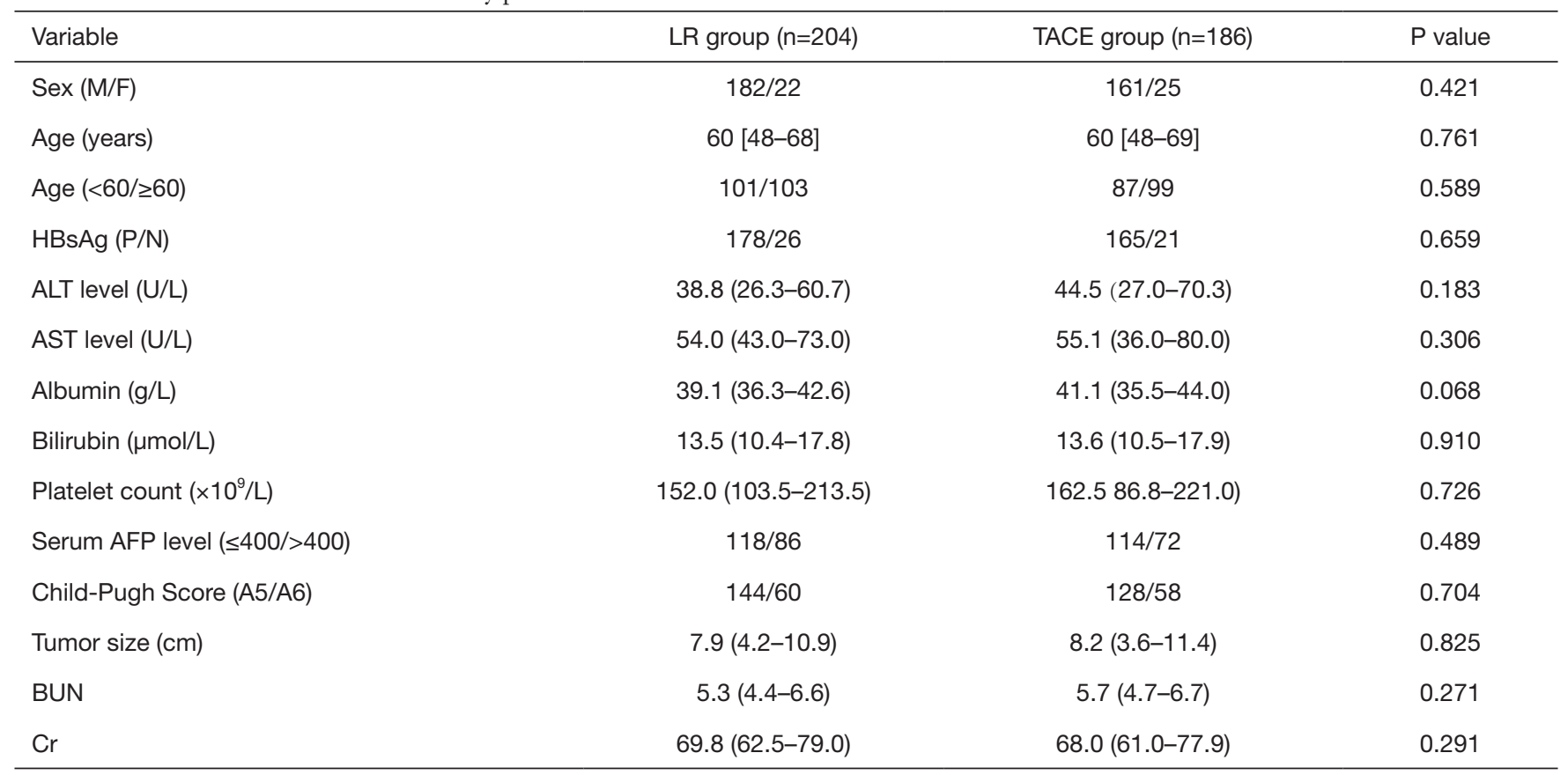

TACE, transarterial chemoembolisation; AFP, alpha fetoprotein; ALBI, albumin-bilirubin; AST, aspartate aminotransferase; ALT, alanine aminotransferase; PT, prothrombin time; SD, standard deviation.

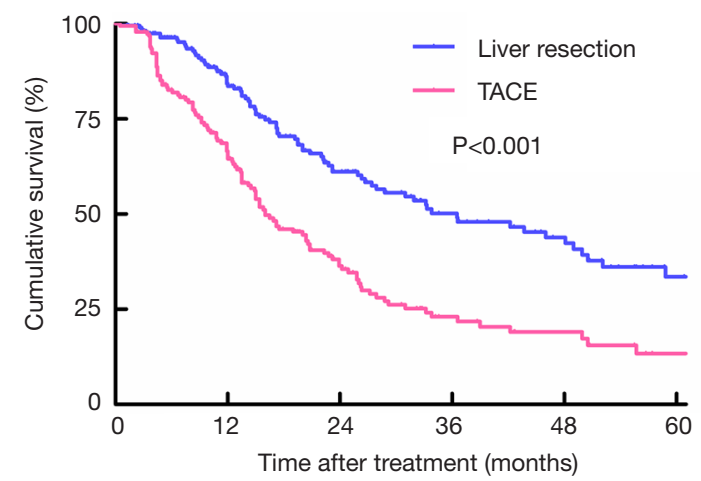

Figure 1 Kaplan-Meier curves for overall survival (OS). TACE, transarterial chemoembolisation.

the independent prognostic factors included tumor size (HR, 1.144; 95\% CI, 1.106-1.184; $\mathrm{P}<0.001$ ), Child-Pugh score (HR, 1.951; 95\% CI, 1.454-1.2.618; $\mathrm{P}<0.001$ ), and treatment method (HR, 2.281; 95\% CI, 1.709-3.046; $\mathrm{P}<0.001)$.

\section{Subgroup analysis}

Tumor size was further stratified into three different groups
Table 2 Univariate analysis of prognostic factors of overall survival

\begin{tabular}{|c|c|c|}
\hline \multirow{2}{*}{ Factors } & \multicolumn{2}{|c|}{ Univariate Cox regression } \\
\hline & Hazard ratio $(95 \% \mathrm{Cl})$ & $P$ value \\
\hline Sex (male) & $1.167(0.755-1.804)$ & 0.486 \\
\hline Age ( $\geq 60$ years) & $0.967(0.732-1.277)$ & 0.811 \\
\hline Tumor size $(\mathrm{cm})$ & 1.135 (1.098-1.173) & $<0.001$ \\
\hline AFP (>400 ng/mL) & $1.188(0.892-1.583)$ & 0.238 \\
\hline Albumin (g/L) & $0.954(0.929-0.980)$ & 0.001 \\
\hline Bilirubin $(\mu \mathrm{mol} / \mathrm{L})$ & $1.035(1.019-1.050)$ & $<0.001$ \\
\hline PLT $\left(\times 10^{9} / \mathrm{L}\right)$ & $0.999(0.997-1.001)$ & 0.118 \\
\hline AST (units/L) & $1.002(0.998-1.004)$ & 0.110 \\
\hline ALT (units/L) & $1.002(0.999-1.005)$ & 0.128 \\
\hline Child-Pugh score & $1.724(1.290-2.305)$ & $<0.001$ \\
\hline Positive HBsAg & $0.841(0.529-1.335)$ & 0.462 \\
\hline BUN & $1.020(0.999-1.040)$ & 0.053 \\
\hline Creatinine & $0.994(0.986-1.003)$ & 0.205 \\
\hline Treatment method & $2.126(1.599-2.828)$ & $<0.001$ \\
\hline
\end{tabular}

AFP, alpha fetoprotein; PLT, platelets; AST, aspartate aminotransferase; ALT, alanine aminotransferase; BUN, blood urea nitrogen. 
Table 3 Multivariate analysis of prognostic factors of overall survival

\begin{tabular}{|c|c|c|c|c|}
\hline Factors & \multicolumn{2}{|c|}{ Multivariate model 1} & \multicolumn{2}{|c|}{ Multivariate model 2} \\
\hline Treatment method & 2.610 (1.935-3.521) & $<0.001$ & $2.281(1.709-3.046)$ & $<0.001$ \\
\hline Albumin (g/L) & $0.950(0.925-0.975)$ & $<0.001$ & - & - \\
\hline Bilirubin ( $\mu \mathrm{mol} / \mathrm{L})$ & $1.044(1.028-1.061)$ & $<0.001$ & - & - \\
\hline Tumor size (cm) & $1.137(1.099-1.177)$ & $<0.001$ & $1.144(1.106-1.184)$ & $<0.001$ \\
\hline
\end{tabular}

Table 4 Subgroup analyses of prognostic factors of overall survival

\begin{tabular}{lccc}
\hline Variables & N (liver resection/TACE) & Median survival (liver resection vs. TACE) & P value \\
\hline Tumor size $(\mathrm{cm})$ & & & \\
& & & \\
3 & $12 / 13$ & $48.100 \pm 7.914$ vs. $31.000 \pm 6.570$ & $<0.001$ \\
$3-5$ & $67 / 47$ & $43.700 \pm 11.804$ vs. $16.500 \pm 1.767$ & $<0.001$ \\
$\geq 5$ & $125 / 126$ & $19.500 \pm 4.127$ vs. $12.000 \pm 2.129$ & $<0.001$ \\
\hline
\end{tabular}

TACE, transarterial chemoembolisation.

to identify whether it influenced the efficacy of HR (Table 4). For patients with a tumor size of $\leq 3 \mathrm{~cm}$, those who underwent liver resection showed a better OS than those treated with TACE $(\log$-rank $\mathrm{P}<0.001)$. For patients with a tumor size of $3-5 \mathrm{~cm}$, there was a significant difference in OS between the two groups (log-rank $\mathrm{P}<0.001)$. Among the patients with a tumor size of $\geq 5 \mathrm{~cm}$, those who underwent liver resection had a better OS than those treated with TACE $(\log -$ rank $\mathrm{P}<0.001)$.

\section{Discussion}

In this retrospective study, we demonstrated that BCLC stage $\mathrm{C}$ patients with PS 1 who had no vascular invasion or extrahepatic spread who underwent liver resection had a significantly better OS compared with similar patients who underwent TACE therapy. Furthermore, treatment method (liver resection $v s$. TACE) was an independent prognostic indicator of better OS, while poorer OS was indicated by the size and number of tumors.

As proposed by the Barcelona Clinic Liver Cancer (BCLC) staging system, sorafenib is considered to be the standard treatment option for HCC patients of BCLC stage C. This is a diverse stage, which includes a wide range of patients with single or multiple factors, such as symptomatic tumors that have an adverse effect on PS (ECOG PS 1-2), vascular invasion (either segmental or portal invasion), or extrahepatic spread (lymph node involvement or metastases). Because of the considerable heterogeneity, a diverse range of prognostic outcomes has been observed in stage $C$ patients under the treatment of sorafenib, for whom OS is still unsatisfactory (21). Multiple previous studies have suggested that patients with vascular invasion treated by surgical resection or TACE showed a better survival outcome compared with those treated with sorafenib (22). In addition, liver resection has been shown to have better efficacy than TACE in BCLC stage A patients, although this has been controversial in previous reports, especially regarding its benefits for patients with only one tumor regardless of its size (2). Additionally, PS, which is applied to assess patient's capability of self-care, is deemed to be a powerful predictive factor with respect to the OS of HCC patients. Treatment modality is widely understood to be highly related to OS in HCC patients. PS, to some extent, can influence treatment decisions. To avoid confounding factors, only patients who had a single tumor with PS 1 and no vascular invasion or extrahepatic spread who underwent liver resection or TACE were enrolled in the present study. Our study showed that liver resection was more effective than TACE in enhancing prognostic survival in these patients.

Univariate and multivariate analyses were conducted 
to reveal the prognostic factors of OS. In line with the findings of previous studies, patients with poor prognosis were associated with high grade of ECOG PS. To avoid collinearity in the multivariable analysis, albumin, bilirubin, and the Child-Pugh score were entered into two different Cox proportional hazards regression models. A relationship was seen between patients with poorer prognosis and larger tumor size and higher bilirubin level, while high albumin level was regarded as an indicator of better OS. Additionally, the different treatment methods became significant independent predictors of OS. Subgroup analysis revealed that both liver resection and TACE were significantly related to OS across the entire subsets, with surgical resection having a better prognostic performance than TACE. This demonstrated that liver resection might be a more effective treatment option for BCLC stage C patients with PS 1 and no vascular invasion or extrahepatic spread who have a single tumor, regardless its size.

However, several limitations of this study need to be discussed. The primary limitation of this study is its retrospective design, which could have introduced information bias. All procedures and administration were conducted by the same experienced team to ensure quality control and to alleviate potential bias. Additionally, this study was carried out at a single center and had a relatively small sample size, which could reduce its representativeness. Further highquality prospective studies with large sample sizes are needed. Finally, the majority of the patients in our study were Chinese with hepatitis B infection as the cause of HCC, whereas the etiologies of HCC in most Western countries are mainly hepatitis $\mathrm{C}$ infection and alcoholic liver disease.

\section{Conclusions}

In conclusion, this retrospective study demonstrated that liver resection could provide a better survival outcome in comparison with TACE and should be suggested as an alternative treatment modality for patients with PS 1 who have only a single tumor and no vascular invasion or extrahepatic spread.

\section{Acknowledgments}

Funding: None.

\section{Footnote}

Conflicts of Interest: All authors have completed the ICMJE uniform disclosure form (available at http://dx.doi. org/10.21037/atm.2020.02.83). LL serves as the unpaid editorial board member of Annals of Translational Medicine from Apr 2020 to Mar 2022. The other authors have no conflicts of interest to declare.

Ethical Statement: The authors are accountable for all aspects of the work in ensuring that questions related to the accuracy or integrity of any part of the work are appropriately investigated and resolved. This investigation was approved by the Clinical Research Ethics Committee of the Tangdu Hospita and performed in adherence with the Declaration of Helsinki.

Open Access Statement: This is an Open Access article distributed in accordance with the Creative Commons Attribution-NonCommercial-NoDerivs 4.0 International License (CC BY-NC-ND 4.0), which permits the noncommercial replication and distribution of the article with the strict proviso that no changes or edits are made and the original work is properly cited (including links to both the formal publication through the relevant DOI and the license). See: https://creativecommons.org/licenses/by-nc-nd/4.0/.

\section{References}

1. Bray F, Ferlay J, Soerjomataram I, et al. Global cancer statistics 2018: GLOBOCAN estimates of incidence and mortality worldwide for 36 cancers in 185 countries. CA Cancer J Clin 2018;68:394-424.

2. European Association for the Study of the Liver. EASL Clinical Practice Guidelines: Management of hepatocellular carcinoma. J Hepatol 2018;69:182-236.

3. Tsilimigras DI, Bagante F, Sahara K, et al. Prognosis After Resection of Barcelona Clinic Liver Cancer (BCLC) Stage 0, A, and B Hepatocellular Carcinoma: A Comprehensive Assessment of the Current BCLC Classification. Ann Surg Oncol 2019;26:3693-700.

4. de Freitas LBR, Longo L, Santos D, et al. Hepatocellular carcinoma staging systems: Hong Kong liver cancer vs Barcelona clinic liver cancer in a Western population. World J Hepatol 2019;11:678-88.

5. Zhong JH, Peng NF, You XM, et al. Tumor stage and primary treatment of hepatocellular carcinoma at a large tertiary hospital in China: A real-world study. Oncotarget 2017;8:18296-302.

6. Cheng AL, Kang YK, Chen Z, et al. Efficacy and safety of sorafenib in patients in the Asia-Pacific region with 
advancedhepatocellular carcinoma: a phase III randomised, double-blind, placebo-controlled trial. Lancet Oncol 2009;10:25-34.

7. Llovet JM, Ricci S, Mazzaferro V, et al. Sorafenib in advanced hepatocellular carcinoma. N Engl J Med 2008;359:378-90.

8. Sun Y, Ji S, Ji H, et al. Clinical efficacy analysis of transcatheter arterial chemoembolization (TACE) combined with radiofrequency ablation (RFA) in primary liver cancer and recurrent liver cancer. J BUON 2019;24:1402-7.

9. Jun BG, Kim SG, Kim YD, et al. Combined therapy of transarterial chemoembolization and stereotactic body radiation therapyversus transarterial chemoembolization for $\leq 5 \mathrm{~cm}$ hepatocellular carcinoma: Propensity score matching analysis. PLoS One 2018;13:e0206381.

10. Zhu H, Ye B, Qiao Z, et al. Hepatectomy combined with sorafenib in patients with intermediate-advanced hepatocellullar carcinoma. J BUON 2019;24:1382-9.

11. Wang JC, Xia AL, Xu Y, et al. Comprehensive treatments for hepatocellular carcinoma with portal vein tumor thrombosis. J Cell Physiol 2019;234:1062-70.

12. Chen ZW, Lin ZY, Chen YP, et al. Clinical efficacy of endovascular radiofrequency ablation in the treatment of portal vein tumor thrombus of primary hepatocellular carcinoma. J Cancer Res Ther 2018;14:145-9.

13. Zhang ZY, Dong KS, Zhang EL, et al. Resection might be a meaningful choice for hepatocellular carcinoma with portal vein thrombosis: A systematic review and metaanalysis. Medicine (Baltimore) 2019;98:e18362.

14. Lv WF, Liu KC, Lu D, et al. Transarterial chemoembolization for hepatocellular carcinoma combined with portal vein tumor thrombosis. Cancer Manag Res 2018;10:4719-26.

Cite this article as: Zhao S, Zhang X, Wang M, Tan K, Dou W, Fan Q, Li H, Du X, Liu L. Identifying optimal candidates for liver resection or transarterial chemoembolisation in patients with unresectable hepatocellular carcinoma. Ann Transl Med 2020;8(9):586. doi: 10.21037/atm.2020.02.83
15. Hsu CY, Lee YH, Hsia CY, et al. Performance status in patients with hepatocellular carcinoma: determinants, prognosticimpact, and ability to improve the Barcelona Clinic Liver Cancer system. Hepatology 2013;57:112-9.

16. Wu J, Li A, Yang J, et al. Efficacy and safety of TACE in combination with sorafenib for the treatment of TACErefractory advanced hepatocellular carcinoma in Chinese patients: a retrospective study. Onco Targets Ther 2017;10:2761-8.

17. Vitale A, Burra P, Frigo AC, et al. Survival benefit of liver resection for patients with hepatocellular carcinoma across differentBarcelona Clinic Liver Cancer stages: a multicentre study. J Hepatol 2015;62:617-24.

18. Facciorusso A, Del Prete V, Antonino M, et al. Serum ferritin as a new prognostic factor in hepatocellular carcinoma patients treated with radiofrequency ablation. J Gastroenterol Hepatol 2014;29:1905-10.

19. Bruix J, Sherman M, and American Association for the Study of Liver Diseases. Management of hepatocellular carcinoma: an update. Hepatology 2011;53:1020-2.

20. European Association For The Study Of The Liver and European Organisation For Research And Treatment Of Cancer. EASL-EORTC clinical practice guidelines: management of hepatocellular carcinoma. J Hepatol 2012;56:908-43.

21. Cho HJ, Kim SS, Kang SY, et al. A Proposal for Modification of the Barcelona Clinic Liver Cancer Staging System Considering the Prognostic Implication of Performance Status. Gut Liver 2019;13:557-68.

22. Peng SY, Wang XA, Huang CY, et al. Better surgical treatment method for hepatocellular carcinoma with portal vein tumor thrombus. World J Gastroenterol 2018;24:4527-35. 